(C) 2008 IEEE. Personal use of this material is permitted. Permission from IEEE must be obtained for all other uses, in any current or future media, including reprinting/republishing this material for advertising or promotional purposes, creating new collective works, for resale or redistribution to servers or lists, or reuse of any copyrighted component of this work in other works. 


\title{
A Subgoal-Guided Force Field Method for Robot Navigation
}

\author{
D. Wang, D. K. Liu, N. M. Kwok, K. J. Waldron
}

\begin{abstract}
The Force Field $\left(\mathrm{F}^{2}\right)$ method is a novel approach to robot motion planning and collision avoidance. This research analyzes some shortcomings of the $F^{2}$ method and then presents the Subgoal-Guided $F^{2}$ method. In the proposed approach, a robot identifies openings in front of the robot in an environment based on sensor data. The midpoints of these openings are determined and selected as subgoal candidates. A cost function is then utilized to evaluate these candidates. One subgoal is then chosen and used by $F^{2}$ method to generate the attractive force which will attracts this robot to the subgoal. The subgoal is continuously updated based on real-time sensor data until the global goal is reached. Simulations are carried out to show the feasibility of the proposed approach.
\end{abstract}

\section{INTRODUCTION}

$\mathrm{T}$ he robot motion planning problem has been extensively studied in the past decades and a variety of techniques have been presented [1]. While a complete review of existing work is not possible due to space limitation, we only introduce some approaches which are closely related to our work.

Potential Field Method (PRM) has been a popular approach for single autonomous mobile robot path planning because of its mathematical simplicity and efficiency [2]. The basic concept of PFM method is to fill a working space with an artificial potential field in which the robot is attracted by the goal and repulsed away by obstacles. Researchers have developed various methods based on the potential field concept. Connolly presented a method using the Laplace's equation to avoid local minima [3]. Masoud proposed a repulsive field which is strictly localized in a robot's vicinity to protect it from collision [4]. In Masoud's method, the repulsive field is generated as the gradient flow of a spherically symmetric scalar force potential field. Ge and Cui developed a potential field method which defines attractive and repulsive potentials by taking into account the relative position and velocity of a robot with respect to obstacles and targets [5]. Borenstein and Koren developed the Vector Field Method (VFH), a method that looks for gaps in locally constructed polar histograms [6]. Further researches led to $\mathrm{VFH}+[7]$ and $\mathrm{VFH}^{*}[8]$. Based on $\mathrm{VFH}+$, An and Wang presented VPH, in which a laser radar is used to detect obstacles and the physical meaning of the vector is taken into

Manuscript received June 16, 2008. This work is supported in part by the ARC Centre of Excellence programme, funded by the Australian Research Council (ARC) and the New South Wales State Government, Australia.

D. Wang, D. K. Liu and N. M. Kwok are with the ARC Centre of Excellence for Autonomous Systems (CAS), Faculty of Engineering, University of Technology, Sydney, (UTS), NSW 2007, Australia. (e-mail: \{Da-Long.Wang, dkliu, ngai.kwok\}@ eng.uts.edu.au).

K. J. Waldron is with the Department of Mechanical Engineering, Stanford University, Stanford, CA, USA (email: kwaldron@stanford.edu) consideration [9]

Simmons suggested the Curvature-Velocity Method $(\mathrm{CVM})$, in which constraints derived from physical limitations on the robot's velocities and accelerations, and from sensor data that indicate the presence of obstacles are treated in motion planning [10]. In the Dynamic Window Approach (CVM), kinematic constrains were taken into account by directly searching the velocity space of a synchro-drive robot [11]. By taking some dynamic constrains into consideration, CVM and DWA reduce the search space greatly. Brock and Khatib extended the DWA to Global Dynamic Window Approach (GDWA), which is applicable to both nonholonomic and holonomic mobile robots and is suitable for unknown and changing environment [12].

The concept of subgoal is widely used in the robot motion planning [13-16]. In [14], the subgoal positions are continuously updated based on sensor data while robots are moving. In a manipulator path planning problem, a global search tries to find a sequence of subgoals and a serial local search conducts local search between subgoals. This method traces back to the global search when the local search fails to find local paths [15]. In [16], long-range sensor data and global information are used to generate an intermediate goal and the short-range sensors are used to guide a robot to this subgoal.

The $\mathrm{F}^{2}$ method is a force field based method for multi-robot path planning and collaboration [17]. Instead of generating potential fields or force fields for the environment and obstacles, a virtual force field is constructed for each robot based on its status, including traveling speed, dimension, priority, location and environmental factors, etc. The force field of a robot is different from those of other robots due to its different status and varies with the robot during its movement. A robot with larger volume, higher travelling speed, or higher task priority than other robots will have a larger force field and preference in collision avoidance. The interaction among the robots' force fields and obstacles provides a feasible and efficient way for multi-robot motion planning and collaboration.

The concept of $\mathrm{F}^{2}$ resembles the PFM to some extent. Both concepts use the repulsive potential/force field to avoid collision with obstacles and the attractive potential/force to guide a robot to its target. But the differences between the $\mathrm{F}^{2}$ and PFM are distinct. The potential field in PFM is generated based on environment information. That is to say, the potential value of a point in potential field is determined by its location with respect to obstacles nearby and the goal. This potential field remains unchanged if the environment does not change. In the $\mathrm{F}^{2}$ method, the repulsive force field of a robot 
TABLE I

PARAMETERS IN $\mathrm{F}^{2}$ METHOD

\begin{tabular}{cl}
\hline \hline Parameters & \multicolumn{1}{c}{ Descriptions } \\
\hline$R_{r}$ & radius of a robot \\
$v_{r}$ & absolute value of a robot's speed \\
$v_{\max }$ & maximum absolute value of a robot's speed \\
$T_{p}$ & a robot's task priority \\
$\theta_{r}$ & angle between a robot's moving direction and the X \\
& coordinate \\
\hline \hline
\end{tabular}

is generated around the robot body, instead of around obstacles as those of potential field based approaches, and is continuously changing during this robot's movement. Collision avoidance is achieved by interaction between this virtual protective field and environment.

The $F^{2}$ method and its variations have been extensively tested with real robots in various environments since they were developed. In this research, we will analyze some revealed shortcomings of the $\mathrm{F}^{2}$ method and then present the Subgoal-Guided $\mathrm{F}^{2}$ method which enhances the performance of the $\mathrm{F}^{2}$ method greatly. This paper is organized as follows. In Section II, we give a review of the $\mathrm{F}^{2}$ method. Section III describes the proposed Subgoal-Guided $\mathrm{F}^{2}$ method. The feasibility of this approach is supported by simulations described in Section IV. Conclusion and future work are given in Section V.

\section{II. $\mathrm{F}^{2} \mathrm{METHOD}$}

In the $\mathrm{F}^{2}$ method, a robot is assumed to travel in a 2-dimensional environment and its location can be precisely known. Each robot is aware of all other robots, such as their locations, speeds, etc. For simplicity of explanation, robots are represented as round discs, but can be easily extended to other complex geometric shapes. For more information regarding the $\mathrm{F}^{2}$ method, see $[18,19]$.

\section{A. Definition of a Force Field}

A force field is a virtual field of repulsive force in the vicinity of a robot when it travels in a working space. The magnitude and orientation of a force field are determined by and vary with the robot's status. This virtual repulsive force diminishes in proportion to the distance from the robot. Parameters in the $\mathrm{F}^{2}$ method are listed in Table I.

We define:

$$
\begin{aligned}
& \theta=\angle\left(\theta_{o}, \theta_{r}\right) \\
& E_{r}=v_{r} /\left(v_{\max } C\right) \\
& D_{\max }=\frac{k E_{r} R_{r}}{1-E_{r} \cos \theta} T_{p} \\
& D_{\text {min }}=\rho_{0} D_{\max }
\end{aligned}
$$

For any point $(x, y)$ in the 2-D space, $\theta$ denotes the relative angle of this point to the robot's orientation (Fig. 1). $C$ is a positive constant which denotes the environment influence to the force field with $C>1 . E_{r}$ is a positive decimal fraction with $0 \leq E_{r}<1 . k$ is a positive multiplier which determines the coverage area of the force field. $D_{\max }$ is the maximum active distance of a robot's force field and $D_{\min }$ is the distance at which this robot has maximum repulsive force. $D_{\max }$ determines how far this robot can affect others in its vicinity. $D_{\min }$ provides a safe distance for the robot to prevent other objects from moving into this area. $\rho_{0}$ is a positive fractional number with $0<\rho_{0}<1$ and heavily influences how close the robot can be safely navigated in the vicinities of obstacles. $T_{p}$ represents the priority of a task which is undertaken by the robot with $T_{p} \geq 1$. Please note that $T_{p}$ is set to 1 for the single robot case.

The repulsive force generated by a robot is defined by:

$$
\begin{aligned}
& \mid \begin{array}{ll}
\left|\vec{F}_{\text {repusive_robot }}\right|= & \text { when } D>D_{\max } \\
P \frac{D_{\max }-D}{D_{\max }-D_{\min }} ; & \text { when } D_{\max } \leq D<D_{\min } \\
F_{\max } ; & \text { when } D<D_{\min }
\end{array}
\end{aligned}
$$

where $D$ is the shortest distance from point $(x, y)$ to the perimeter of the robot. $P$ is a positive constant scalar which determines the magnitude of the repulsive force. When $D$ changes from $D_{\min }$ to $D_{\max }$, the magnitude of the repulsive force changes from $P$ to 0 gradually. $F_{\max }$ is the maximum repulsive force which will cause the maximum deceleration on the robot. $P$ and $F_{\max }$ should be selected based on the robot's characteristics, with $F_{\max }>>P$. Equation (5) shows that the magnitude of repulsive force varies with distance. To represent the force field in contours, we further define:

$$
\rho=D / D_{\max }
$$

Then (5) can be re-presented in an alternative form as:

$$
\begin{aligned}
& \left|\vec{F}_{\text {repusive_robot }}\right|= \\
& \begin{cases}0 ; & \text { when } \rho>1 \\
P \frac{1-\rho}{1-\rho_{0}} ; & \text { when } \rho_{0} \leq \rho<1 \\
F_{\max } ; & \text { when } \rho<\rho_{0}\end{cases}
\end{aligned}
$$

When $\rho$ changes from $\rho_{0}$ to 1 , the magnitude of the repulsive force changes from $P$ to 0 .

\section{B. Attractive Force}

When a robot is allocated a particular task, for example, traveling from start point $\left(X_{\text {start }}, Y_{\text {start }}\right)$ to goal point $\left(X_{\text {goal }}\right.$, $\left.Y_{\text {goal }}\right)$, a virtual attractive force which attracts the robot from the start point to the goal point is generated.

$$
\left|\vec{F}_{\text {field }}\right|=Q
$$

where $Q$ is a positive constant scalar which determines the magnitude of the attractive force. The attractive force directs the robot to the goal point from the centre of the robot and can be assumed to be a constant. It drives a robot to its destination $\left(X_{\text {goal }}, Y_{\text {goal }}\right)$. 


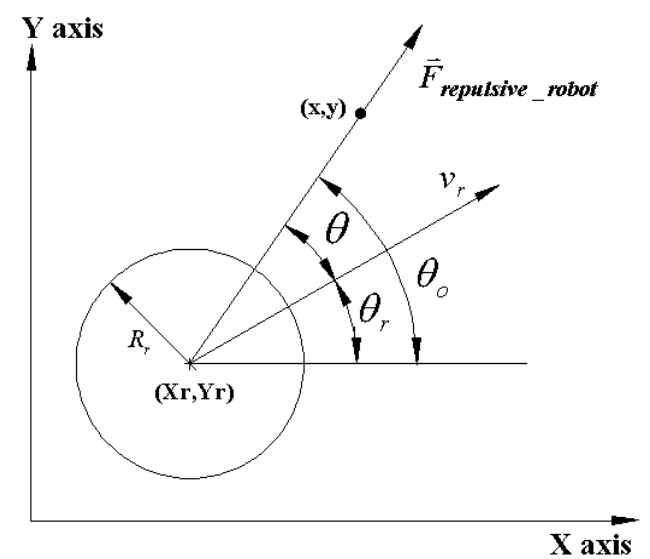

Fig. 1 Illustration of a robot's parameters

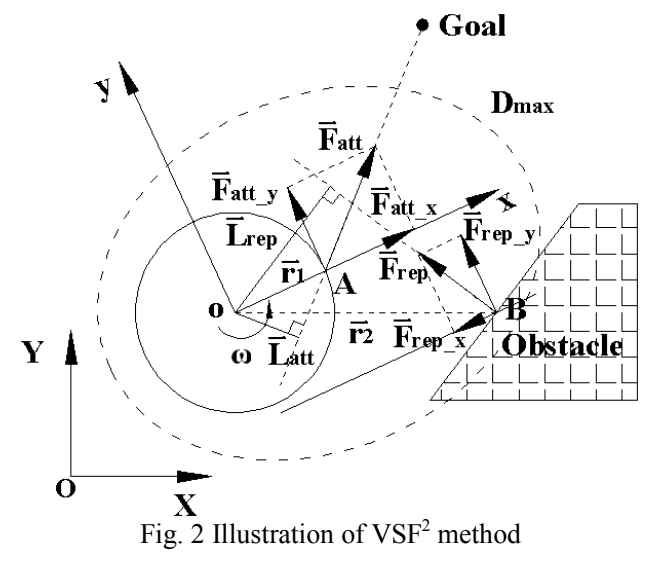

\section{Reaction Force}

When a robot approaches an obstacle or other robots' force fields, its force field is suppressed and the robot is repelled away by the virtual reaction force from obstacles or other robots. This reaction force can be obtained from:

$$
\left|\vec{F}_{\text {rep }}\right|=\left|\vec{F}_{\text {repulsive_robot }}\right|
$$

where $\vec{F}_{\text {repulsive_robot }}$ is the virtual repulsive force generated by this robot at the interaction point $(x, y)$ in the 2-D space. The reaction force is a vector. Its direction is defined as the normal line of the interaction contour with respect to the interaction point.

\section{D. $\quad$ SSF $F^{2}$ Method}

The Variable Speed Force Field method $\left(\mathrm{VSF}^{2}\right)$ is an approach using the concept of the $\mathrm{F}^{2}$ method for multi-robot motion planning and collision avoidance. This section introduces the $\mathrm{VSF}^{2}$ briefly. For more details, refer to [17].

Define a fixed reference frame $O(X, Y)$ and a moving reference frame $o(x, y)$ attached to the robot's body. Let $\dot{x}, \dot{y}$ be the longitudinal and lateral velocity of the robot in frame $o$ $(x, y)$, the absolute velocities $\dot{X}, \dot{Y}$ in the fixed reference frame $O(X, Y)$ are:

$$
\left[\begin{array}{c}
\dot{X} \\
\dot{Y}
\end{array}\right]=\left[\begin{array}{cc}
\cos \theta_{r} & -\sin \theta_{r} \\
\sin \theta_{r} & \cos \theta_{r}
\end{array}\right]\left[\begin{array}{c}
\dot{x} \\
\dot{y}
\end{array}\right]=R\left(\theta_{r}\right)\left[\begin{array}{c}
\dot{x} \\
\dot{y}
\end{array}\right]
$$

$$
\left[\begin{array}{c}
\ddot{X} \\
\ddot{Y}
\end{array}\right]=R\left(\theta_{r}\right)\left[\begin{array}{l}
a_{x} \\
a_{y}
\end{array}\right]
$$

where $a_{x}, a_{y}$ are the accelerations expressed in the moving frame $o$.

For a robot of mass $m$ and inertia $I$ about its centre of mass, equations of motions in the frame $o$ are:

$$
\begin{aligned}
& m a_{x}=\vec{F}_{\text {att_x }}+\sum \vec{F}_{\text {rep_x }} x \\
& m a_{y}=\vec{F}_{\text {att_y }}+\sum \vec{F}_{\text {rep_y }} y \\
& I \dot{\omega}=M_{\text {att }}+\sum M_{\text {rep }}
\end{aligned}
$$

where $\vec{F}_{\text {att_x }}, \vec{F}_{\text {att_y } y}$ are the components of the virtual attractive force along the longitudinal direction and the lateral direction respectively. $\vec{F}_{\text {rep_ }}, \vec{F}_{\text {rep }} y$ are the components of the virtual repulsive forces along the longitudinal direction and the lateral direction respectively (see Fig. 2). $\omega$ is the angular velocity of this robot about its center of mass in frame $o$. $M_{\text {att }}$ and $M_{\text {rep }}$ are the moments generated by the virtual attractive force and repulsive force respectively. The forces and moment are given by:

$$
\begin{aligned}
& M_{\text {att }}=\vec{r}_{1} \times \vec{F}_{\text {att }} \\
& M_{\text {rep }}=\vec{r}_{2} \times \vec{F}_{r e p}
\end{aligned}
$$

where $\vec{r}_{1}$ is the distance from the center of robot $o$ to the attractive force $\left(\vec{F}_{\text {att }}\right)$ acting point $A . \vec{r}_{2}$ is the distance from the center of robot to the repulsive force $\left(\vec{F}_{r e p}\right)$ acting point $B$.

Please note that we assume the attractive force acts on the robot's front edge $A$, which is the interaction of $x$ coordinate and a robot's body (see Fig. 2).

It should be noted that a robot needs to satisfy the limitations of translational speed $\left(v_{r}\right)$, translational acceleration $\left(\dot{v}_{r}\right)$, angular speed $(\omega)$ and angular acceleration $(\dot{\omega})$. These constraints are:

$$
\begin{aligned}
& -v_{\text {max }} \leq v_{r} \leq v_{\text {max }} \\
& -a_{\text {max }} \leq \dot{v}_{r} \leq a_{\max } \\
& -\omega_{\max } \leq \omega \leq \omega_{\max } \\
& -\dot{\omega}_{\max } \leq \dot{\omega} \leq \dot{\omega}_{\max }
\end{aligned}
$$

These parameters should be chosen on the basis of the robot's dynamic and kinematics characteristics.

\section{METHODOLOGY}

This section introduces the proposed Subgoal-Guided $\mathrm{F}^{2}$ method. Although the discussions and simulations in this paper are built upon the $\mathrm{VSF}^{2}$ method, they are still applicable to other $\mathrm{F}^{2}$ based methods. We will start from analyzing the shortcomings of $\mathrm{F}^{2}$ method and then introduce the proposed Subgoal-Guided $\mathrm{F}^{2}$ method in detail.
Differentiation with respect the time gives 


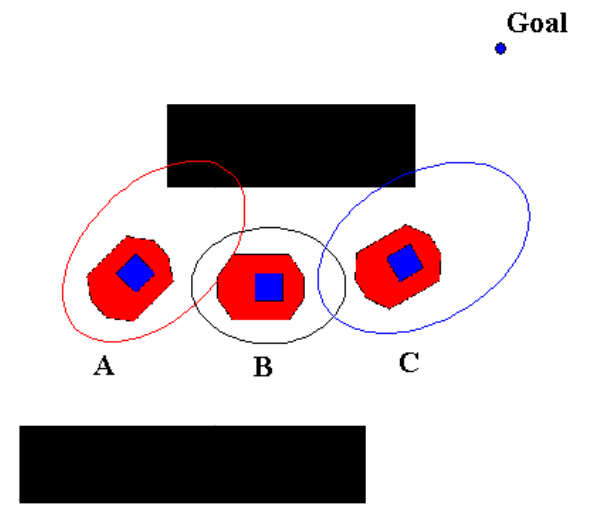

Fig. 3 A problematic case

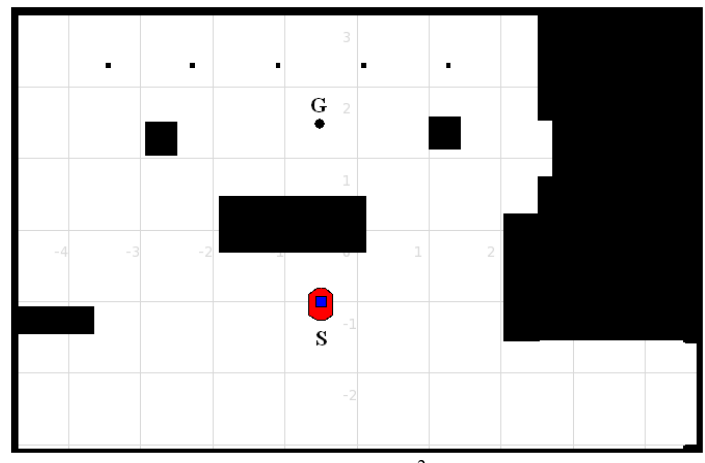

Fig. 4 A local minimum for $\mathrm{F}^{2}$ method and PFM

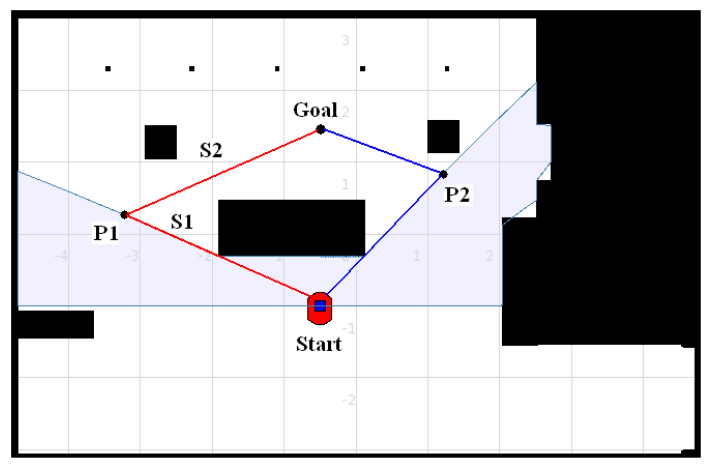

Fig. 5 Illustration of subgoals

\section{A. Motivation}

Figure 3 shows a problematic case of the $\mathrm{F}^{2}$ method. Let an obstacle be located between a robot and its goal position. The three red patches show a robot's several locations on its way towards the goal. The blue patches show the positions of laser sensor onboard. According to the $\mathrm{F}^{2}$ method, the robot is attracted by the attractive force from the goal point and repelled away by repulsive forces from the obstacles. Since a obstacle is on the way of this robot, the robot goes towards the obstacle and will be repelled away when the repulsive force is large enough (location A). Because of the attractive force, the robot turns back to its previous (location B) and will be repulsed away by the obstacle again (location C). This "go towards obstacles" behavior causes the zigzag like movements and oscillations of speed and heading of the robot.
Figure 4 shows a local minimum case of $F^{2}$ method. An obstacle is located between its start point and goal point. This is a local minima problem for both Potential Field Method and $\mathrm{F}^{2}$ method. The attractive force from the goal point and repulsive force from the obstacle are opposite and collinear, which causes the robot to stop at the point where their absolute values are equal (in equilibrium).

\section{B. Subgoal-based Approach}

Based on the analyses above, we introduce subgoals into the $\mathrm{F}^{2}$ method. In the scenarios considered in this paper, a robot is equipped with a laser sensor. Based on the returned range data, a robot identifies openings in front of it. The midpoints of these openings are selected as subgoal candidates. A heuristic function is then utilized to evaluate these candidates.

Figure 5 shows the laser range of the case in Fig. 4. Two openings are found by the laser sensor and their midpoints are denoted by $\mathrm{P}_{1}$ and $\mathrm{P}_{2}$. These midpoints are selected as subgoal candidates. From Fig. 5, it seems that the robot will collide with obstacle edges if it goes directly to subgoals. But this will not really occur since the robot is protected by its repulsive force field while moving.

The cost function applied by the robot to evaluate subgoals is defined as a sum of two functions: the distance from robot to a subgoal candidate, which can be estimated from the sensor reading (denoted by $\mathrm{S}_{1}$ ), and the distance from a subgoal candidate to the global goal (denoted by $\mathrm{S}_{2}$ ), which can be estimated based on the knowledge of the global goal position:

$$
f=k_{1} S_{1}+k_{2} S_{2}
$$

The subgoal candidate with lowest cost $f$ will be selected as the current subgoal. By tuning weighing factors $k_{1}$ and $k_{2}$, different subgoals may be selected. For example, a robot may choose to go to an opening which is close to its final destination (with small $\mathrm{S}_{2}$ ) or tend to go to an opening which is near to its current location (with small $\mathrm{S}_{1}$ ).

When a subgoal is determined and the final destination is not in the sensor's range, an attractive force is generated from this subgoal, instead of from the final destination, which will attractive this robot to the subgoal. Please note that the subgoal is continuously changed and updated based on sensor data when a robot is moving. When the final destination is found within the sensor's range, the robot will go to it directly.

\section{SIMULATIONS}

This section presents some simulations carried out using the Player/Stage platform [20]. A robot is supposed to travel from its start point to a goal point in an indoor environment. This robot is equipped with a laser sensor and an Adaptive Monte-Carlo Localization driver $(\mathrm{amcl})$ is utilized to determine its locations. The update threshold of amcl driver is set to 10 degrees or $5 \mathrm{~cm}$. Some robot parameters are selected based on those of a pioneer robot [21]. In all simulations, $k_{I}$ and $k_{2}$ are set to 1 . 


\section{A. Case 1}

In this simulation, we consider the local minimum case shown in Fig. 4. A robot is supposed to travel from $(-0.5,-1)$ to $(-0.5,1.5)$. An obstacle is located between the robot and its destination. Figure 5 gives a snapshot when the robot is about to move. Two subgoal candidates here are $\mathrm{P}_{1}$ and $\mathrm{P}_{2} . \mathrm{P}_{2}$ is selected as current subgoal according to Equation (21). The resultant path is shown in Fig.6. Figure 7 gives the robot's orientation determined by the localizer during its movement.

\section{B. Case 2}

The map used in this simulation is shown in Fig. 8. A robot is supposed to travel from the start point at $(-3,-2)$ to the destination at $(1,1.5)$. Figure 8 gives a snapshot when the robot is about to move. Six subgoal candidates are found (denoted by $\mathrm{P}_{1}, \mathrm{P}_{2}, \mathrm{P}_{3}, \mathrm{P}_{4}, \mathrm{P}_{5}, \mathrm{P}_{6}$ ) here. The resultant path is shown in Fig. 9. The robot's orientation given by localizer is shown in Fig. 10.

\section{Case 3}

In Case 2, if the environment changes, for example, the lower corridor is blocked as shown in Fig. 11, the subgoal candidates found are depicted as $\mathrm{P}_{1}$ to $\mathrm{P}_{4}$. In this case, $\mathrm{P}_{2}$ ( also $\mathrm{P}_{4}$ in Case 2) is selected as current subgoal. The resultant path is shown in Fig. 12 and corresponding orientation given by localizer is shown in Fig. 13.

\section{Discussions}

In the simulations, the proposed Subgoal-Guided $\mathrm{F}^{2}$ method is tested in various cases. Case 1 shows that Subgoal-Guided $\mathrm{F}^{2}$ method performs better than previous $\mathrm{F}^{2}$ originated methods in respect of solving local minima problem. Since the subgoal is continuously updated based on sensor data, the Subgoal-Guided $\mathrm{F}^{2}$ method is applicable in changing environment (as in Case 2 and Case 3). In all simulations above, the resultant paths are smooth and near optimal.

\section{CONCLUSIONS AND FUTURE WORK}

In this paper, we have presented a novel Subgoal-Guided $\mathrm{F}^{2}$ method, which improves the performance of $\mathrm{F}^{2}$ method greatly. The feasibility of the proposed approach is demonstrated by simulations carried out in the Player/Stage platform.

The subgoal in this paper is selected based on sensor data and used as temporary guidance when the global goal is not in view. Therefore the Subgoal-Guided $\mathrm{F}^{2}$ method is still regarded as a local planner as $\mathrm{F}^{2}$ method. But the subgoal can also be given by a global planner or through communications, which will broaden the usability of $\mathrm{F}^{2}$ method considerably.

\section{ACKNOWLEDGMENT}

This work is supported in part by the ARC Centre of Excellence programme, funded by the Australian Research Council (ARC) and the New South Wales State Government, Australia.

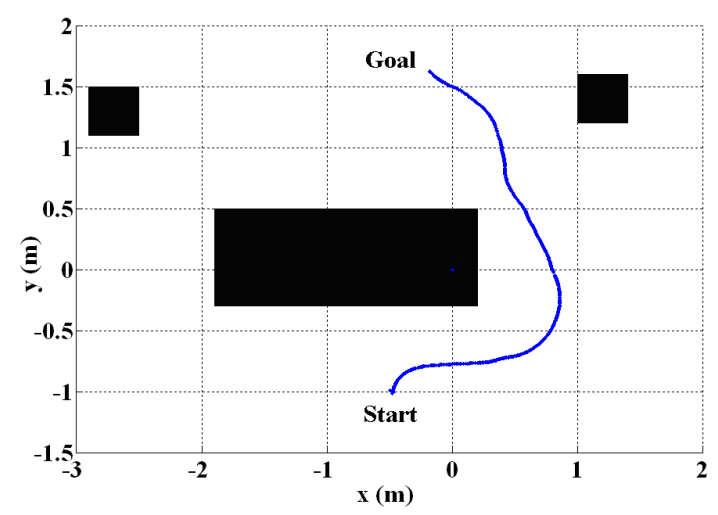

Fig. 6 Case 1: resultant path (dimensions are in $\mathrm{m}$ )

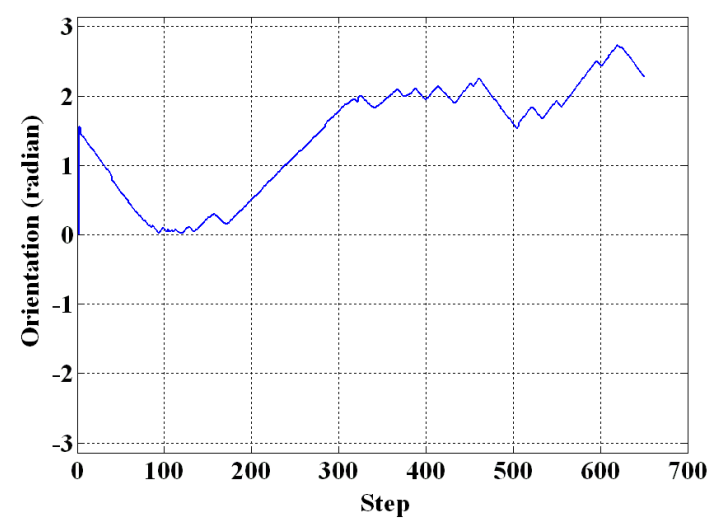

Fig. 7 Case 1: orientation (in radian) traced against simulation time

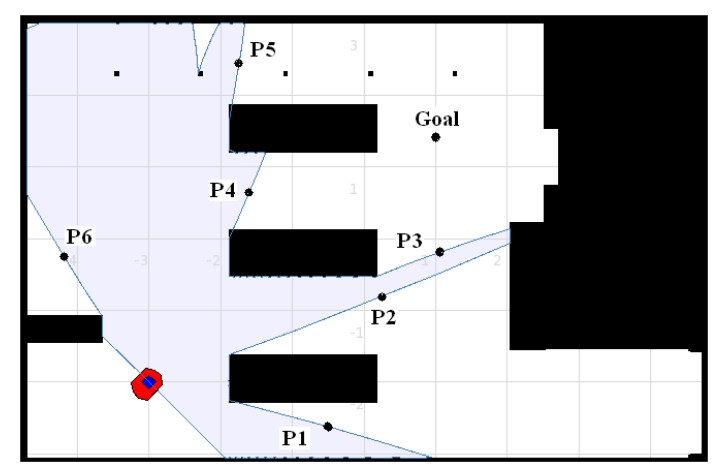

Fig. 8 Case 2: map

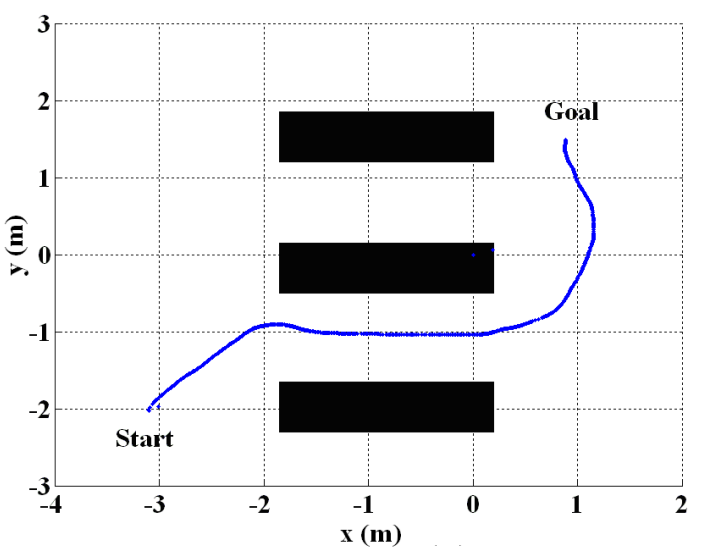

Fig. 9 Case 2: resultant path (dimensions are in $\mathrm{m}$ ) 


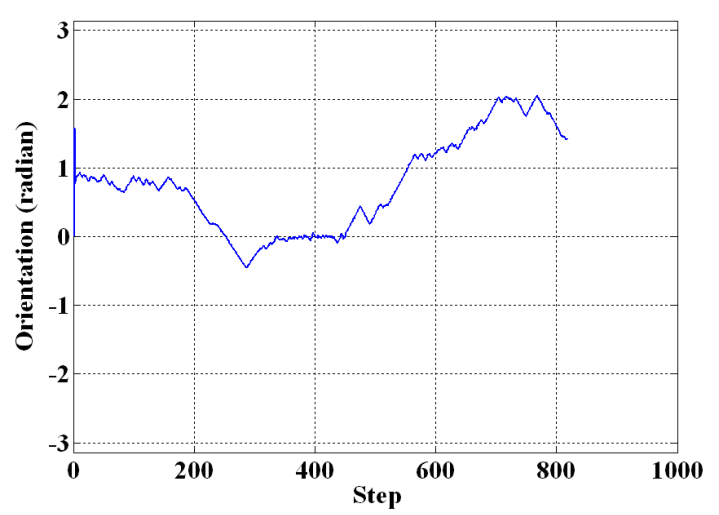

Fig. 10 Case 2: orientation (in radian)

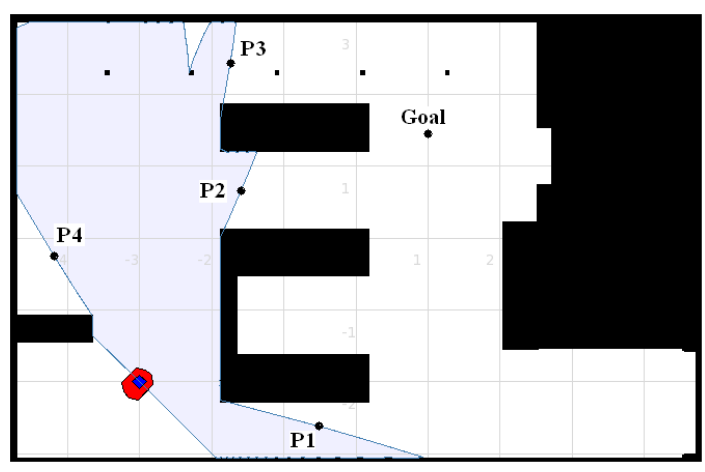

Fig. 11 Case 3: subgoals identified

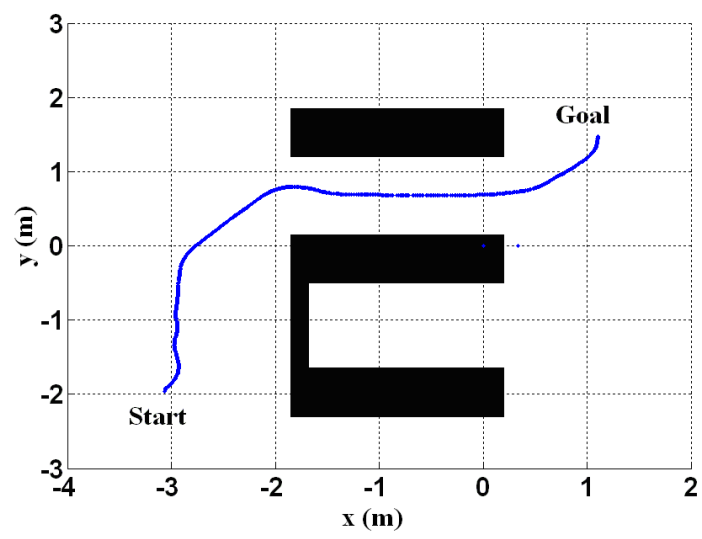

Fig. 12 Case 3: resultant path (dimensions are in $\mathrm{m}$ )

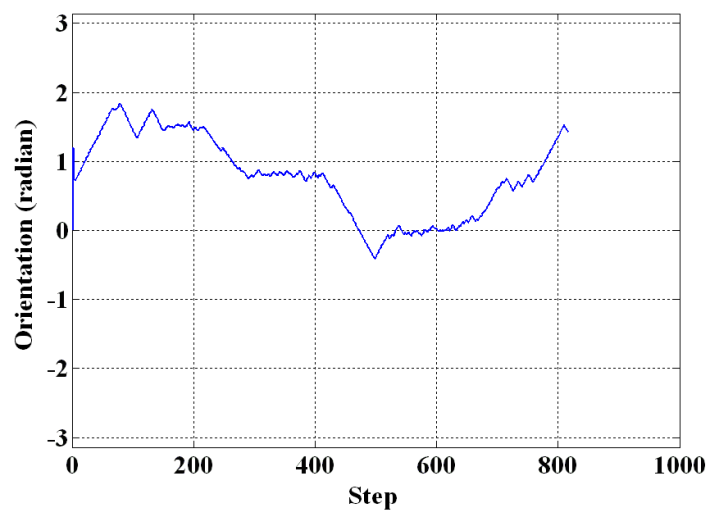

Fig. 13 Case 3: orientation (in radian)

\section{REFERENCE}

[1] J.-C. Latombe, "Robot Motion Planning," Kluwer Academic Publishers, Boston, 1991.

[2] O. Khatib, "Real-time obstacle avoidance for manipulators and mobile robots," in Proceeding of IEEE International Conference on Robotics and Automation, 1985, pp. 500-505.

[3] C. I. Connolly, "Harmonic functions and collision probabilities," in Proceeding of IEEE International Conference on Robotics and Automation, 1994, pp. 3015-3019, vol.4.

[4] A. A. Masoud, "Using hybird vector-harmonic potential fields for multi-robot, multi-target navigation in a stationary environment," in Proceedings of the IEEE International Conference on Robotics and Automation, Minneapolis, Minnesota, 1996, pp. 3564-3571.

[5] S. S. Ge and Y. J. Cui, "Dynamic motion planning for mobile robots using potential field method," Autonomous Robots, vol. 13, pp. 207-222, 2002.

[6] J. Borenstein and Y. Koren, "The vector field histogram-fast obstacle avoidance for mobile robots," IEEE Transactions on Robotics and Automation, vol. 7, pp. 278-288, 1991.

[7] I. Ulrich and J. Borenstein, "VFH+: reliable obstacle avoidance for fast mobile robots," in Proceedings of IEEE International Conference on Robotics and Automation, 1998, vol. 2, pp. 1572-1577.

[8] I. Ulrich and J. Borenstein, "VFH*: local obstacle avoidance with look-ahead verification," in Proceedings of IEEE International Conference on Robotics and Automation (ICRA '00), 2000, vol. 3, pp. 2505-2511

[9] D. An and H. Wang, "VPH: a new laser radar based obstacle avoidance method for intelligent mobile robots," in Proceedings of Fifth World Congress on Intelligent Control and Automation (WCICA 2004), 2004, pp. 4681-4685, vol.5.

[10]R. Simmons, "The curvature-velocity method for local obstacle avoidance," in Proceedings of IEEE International Conference on Robotics and Automation, 1996, pp. 3375-3382, vol.4.

[11]D. Fox, W. Burgard, and S. Thrun, "The dynamic window approach to collision avoidance," IEEE Robotics \& Automation Magazine, vol. 4, pp. 23-33, 1997.

[12]O. Brock and O. Khatib, "High-speed navigation using the global dynamic window approach," in Proceedings of IEEE International Conference on Robotics and Automation, 1999, pp. 341-346, vol.1.

[13]C. W. Warren, "A vector based approach to robot path planning," in Proceedings of IEEE International Conference on Robotics and Automation, 1991, pp. 1021-1026 vol.2.

[14]B. H. Krogh and D. Feng, "Dynamic generation of subgoals for autonomous mobile robots using local feedback information," IEEE Transactions on Automatic Control, vol. 34, pp. 483-493, 1989.

[15]S. Ando, "A fast collision-free path planning method for a general robot manipulator," in Proceedings of. IEEE International Conference on Robotics and Automation, 2003, pp. 2871-2877 vol.2.

[16]Y. Xiaoyu, M. Moallem, and R. V. Patel, "A layered goal-oriented fuzzy motion planning strategy for mobile robot navigation," IEEE Transactions on Systems, Man, and Cybernetics, Part B, vol. 35, pp. 1214-1224, 2005.

[17]D. Wang, D. Liu, and G. Dissanayake, "A variable speed force field method for multi-robot collaboration," in Proceedings of IEEE/RSJ International Conference on Intelligent Robots and Systems, Beijing, China, 2006, pp. 2697-2702.

[18]D. K. Liu, D. Wang, and G. Dissanayake, "A force field method based multi-robot collaboration," in Proceeding of the IEEE International Conference on Robotics, Autonomous \& Mechatronics, Bangkok, Thailand, 2006, pp. 662-667.

[19]D. L. Wang, D. K. Liu, X. Wu, and K. C. Tan, "A force field method for robot navigation," in Proceeding of the Third International Conference on Computational Intelligence, Robotics and Autonomous Systems, 2005, pp. 662-667

[20]B. P. Gerkey, R. T. Vaughan and A. Howard, "The Player/Stage Project: Tools for Multi-Robot and Distributed Sensor Systems," in Proceeding of the 11th International Conference on Advanced Robotics (ICAR 2003), Coimbra, Portugal, 2003, pp. 317-323.

[21]"http://www.activrobots.com/ROBOTS/specs.html." 